\title{
The use of a newspaper insertion to promote DIY testing of vision in India
}

G V S Murthy, Sanjeev Kumar Gupta, Vijay Kumar Dada, Tara Dutt Pant, Chibber Savita, Lalit Sanga, John Neena

\begin{abstract}
Background-The mass media have the potential to motivate people to participate in self appraisal of their own health status. An innovative communication package was designed to help people to examine vision at home. The impact of publishing the "do it yourself" (DIY) kit in Indian newspapers was evaluated.

Methods-A pretested bilingual vision testing kit was published in three newspapers. The kit comprised four tumbling Es corresponding to 6/12 line of Snellen's optotypes. Directions on using the kit were enclosed. 3-7 days after publication of the kit, a telephone survey of newspaper readers was undertaken to evaluate the impact and cost effectiveness.

Results-603 people were contacted over the telephone. $125(20.73 \%)$ subscribed to the newspaper carrying the DIY insertion. $43.2 \%$ (54) noticed the insertion of which $88.89 \%$ (48) read the enclosed instructions carefully. $58.33 \%$ respondents felt sufficiently motivated to contact an ophthalmologist. Graduates had a 3.83 times higher probability of reading the communication insertion compared with others. Differences in relation to other demographic variables were not statistically significant.
\end{abstract}

Conclusions-Newspapers are an excellent medium for communicating self appraisal kits for vision testing. The medium is cost effective and has significant reach in the urban agglomerates of India. (Br f Ophthalmol 2001;85:952-955)

Dr Rajendra Prasad Centre for Ophthalmic Sciences, All India

Institute for Medical

Sciences, New Delhi, India

G V S Murthy

S K Gupta

V K Dada

T D Pant

C Savita

L Sanga

J Neena

Correspondence to:

Dr G V S Murthy, Associate

Professor, Community

Ophthalmology Section, Dr

RP Centre for Ophthalmic

Sciences, AIIMS, Ansari

Nagar, New Delhi, India

110029

comoph@ndf.vsnl.net.in

Accepted for publication 14 February 2001
There have been tremendous advances in recent years in the innovative use of communication to address public health problems. ${ }^{1}$ Experiences gleaned from no smoking campaigns, seatbelt safety crusades, dental hygiene programmes, and other health promotion efforts have shown that social marketing programmes can lead to a broad based behaviour change. ${ }^{2}$ Mass media have been extensively used in promoting such a behavioural change. Studies have documented that newspapers are an important channel to communicate messages, especially in the urban areas. ${ }^{3}$ India is not only the world's most populous country but also publishes the world's largest number of newspapers and magazines. ${ }^{4}$

In spite of the great potential of mass media in communicating innovative ideas to the lay public, it has not been used to good advantage in increasing community participation or self care in eye care, especially so in developing countries like India. The present study reports on the impact of an innovative strategy for testing distance vision, after a social marketing campaign in the English and vernacular press in India, in May and June 2000, on how to test vision at home.

\section{Material and methods}

A bilingual insertion on how to test distance vision at home was designed by the community ophthalmology section of the Dr Rajendra Prasad Centre for Ophthalmic Sciences, All India Institute for Medical Sciences, New Delhi, India (Fig 1). Four tumbling Es corresponding to the $6 / 12$ line of the standard $\mathrm{E}$ chart, were used for testing visual acuity. Directions for use of the "do it yourself" (DIY) vision testing kit were included in the newspaper insertion in both Hindi and English. The insertion was to be used from a distance of 6 metres. The 6/12 line was chosen because readers of newspapers in India are predominantly urban who would not wait till their vision is severely impaired to seek advice. This is because the occupational status of the urban population demands better visual status than the predominantly agriculture based occupations in rural India. This cut off would therefore be more appropriate for an urban audience in India. It was felt that a $6 / 9$ cut off would have a lower specificity and therefore lead to a "scare" of poor vision among the newspaper readers. It would also push a large number of "normally sighted" individuals into an eye care facility for an unwarranted examination. At the same time, though a $6 / 18$ cut off has a higher specificity, it would miss many people with an uncorrected refractive error and those in the early stages of sight threatening conditions like cataract.

The prototype was pretested among out patients attending the outpatient clinics at the Dr Rajendra Prasad Centre for Ophthalmic Sciences and necessary modifications were incorporated before releasing the version in the newspapers. During the pilot phase, the comprehensibility of the proposed insertion was also evaluated by asking respondents what they understood after reading the matter. Special attention was paid to the accuracy of the testing procedure by examining the vision of all the respondents, after they completed a self appraisal. Vision testing was done by trained optometrists. The Department for International Development (DFID) sponsored the communication insertion as part of a larger community ophthalmology project. 
Test your vision at home

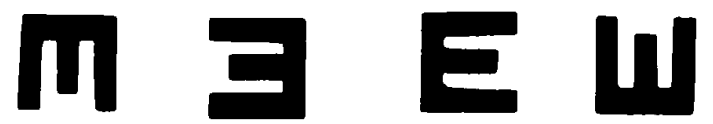

Directions

1. Cut this along the dotted line and paste it as a whole on a hard board with cellotape.

2. Ask someone around you (he lshe may be your family member, friend. partner) to take your vision.

3. Keep distance of 6 metres (20 feet) between you and your friend.

4. Helshe should stand in a well-lit place for taking vision

5. Helshe should hold this card in front of him Iher of chest level.

b. In case you weor glasses for distance, you must weor them while testing your vision.

7. Close your left eye with the paim of your left hand. Do not press the eye.

3. Look ot the card and see the direction of arms of the first $E$ with your right eye.

3. One by one, identify all the 4 Es, indicating the direction of the arms.

10. Repeat the cbove with your left eye, by turning the board upside down.

11. If you cannot identify at least 3 Es with each eye. you must consult an eye doctor.

It is atrieable to consult en eve dactor ance o pou

The sise of the aboue $E$ conforms to $6 / 12$ of the sinellen's chert

Developed by Community Ophthalmology section

DQ RAJENDRA PRASAD CENTRE FOR OPHTHALMIC SCIENCES

All India Institute of Medical Sciences, New Delhi - 110029

Funded by: Depertment for International Development - India

Figure 1 Prototype vision testing kit insertion in newspapers.

The proactive insertion, measuring $22 \mathrm{~cm} \times$ $19 \mathrm{~cm}$, was carried in two English and one Hindi national dailies published from New Delhi. The newspapers were The Times of India, The Hindustan Times (English), and the Dainik Hindustan (Hindi). The do it yourself instructions were carried in these newspapers on four separate days on the 10 (The Times of India), 11 (Dainik Hindustan), and 17 (The Hindustan Times) May, and 18 June 2000 (The Hindustan Times). The total cost of the campaign was Indian rupees 247886 ( US\$ 5500).

From the third day to the seventh day after the insertion appeared in The Hindustan Times on the 18 June 2000, a randomly selected population of telephone users was contacted over telephone and information solicited on the DIY insertion. Telephone ownership is predominantly urban in India with $55 \%$ of telephone owners located in urban areas. The density of telephones is 1.5 telephones per 100 people. Thus, telephone ownership and newspaper readership are more common among the urban middle and upper middle socioeconomic strata in India.

Telephone numbers were randomly generated from the electronic version of the New Delhi telephone directory; 800 numbers were generated after stratifying into the eight area telephone exchange zones (100 per zone). Data on the age, sex, educational level, occupation, reactions to the campaign and follow up action were collected from all respondents who could be contacted, and who were subscribing to The Hindustan Times. Each telephone number was dialled three times at different times of the day on three consecutive days, if there was no answer on the first attempt. If the number could not be contacted even after three attempts, it was categorised as lost response/absent family.

Data were entered in a database designed in EPI-INFO (version 6.0) and all data were transferred to STATA 6.0 for analysis, using STAT TRANSFER 5.0 software packages.

\section{Results}

Among the 800 telephone numbers that were randomly generated, contact could be established with 603 numbers (75.4\%). Among the remaining 197 numbers, 63 numbers had changed and the new numbers were not available. In the case of the remaining 134 numbers, there was either a constant ring or a constant engaged tone. This could either mean that the telephone may have been malfunctioning or there was nobody available to answer the call. Such numbers were classified as lost response/absent family. In two instances among the above, answerphones were switched on and no direct contact could be established.

Among the 603 people who could be contacted, only 125 (20.73\%) subscribed to The Hindustan Times which carried the DIY insertion of 18 June 2000. Most respondents answered all the questions, but $11.2 \%$ (14) respondents refused to answer some details. An additional six $(4.8 \%)$ respondents either slammed the phone down or stated that they did not want to be disturbed.

The mean age of respondents who consented to mention their age (107) was 37.04 years (SD 13.59). The minimum age of respondents was 15 years and the maximum age was 72 years; $38.4 \%$ respondents were aged $41+$ years (Table 1 ). Half the respondents who volunteered information on their sex were females; $61.6 \%$ respondents were either graduates or postgraduates; $36 \%$ respondents were engaged in household work, as they were not gainfully employed.

Of the 125 people who subscribed to The Hindustan Times, 43.2\% (54) stated that they

Table 1 Basic demographic characteristics of surveyed population

\begin{tabular}{lll}
\hline Parameters & Frequency & $\%$ \\
\hline Age profile & & \\
$\quad \leqslant 25$ years & 24 & 19.2 \\
26-40 years & 33 & 26.4 \\
41+ years & 48 & 38.4 \\
$\quad$ Age not recorded/refused & 20 & 16.0 \\
Sex & & \\
$\quad$ Male & 45 & 36.0 \\
Female & 63 & 50.4 \\
Sex not recorded/refused & 17 & 13.6 \\
Educational level & & \\
$\quad$ Graduate & 31 & 24.8 \\
$\quad$ Graduate + & 77 & 61.6 \\
$\quad$ Literacy not recorded/refused & 17 & 13.6 \\
Occupation & & \\
$\quad$ Household work & 45 & 36.0 \\
$\quad$ Students & 18 & 14.4 \\
$\quad$ Business enterprise/self employed & 24 & 19.2 \\
$\quad$ Serving in senior positions & 23 & 18.4 \\
$\quad$ Occupation not recorded/refused & 15 & 12.0 \\
\hline
\end{tabular}


Table 2 Salient observations of readership telephone survey

\begin{tabular}{lll}
\hline Description of readership survey & Frequency & $\%$ \\
\hline Noticed communication insertion in paper & $54 / 125$ & 43.2 \\
Read communication insertion in paper carefully & $48 / 54$ & 88.89 \\
Followed instructions given in the insertion & $43 / 48$ & 89.58 \\
<3 Es seen by respondent with any eye & $6 / 43$ & 13.95 \\
Seen or planning to see an ophthalmologist & $28 / 48$ & 58.33 \\
\hline
\end{tabular}

had noticed the DIY insertion in the newspaper (Table 2). Among them, 88.89\% (48) stated that they read the instructions carefully, and $89.58 \%$ (43) stated that they followed the instructions for examining their vision; $13.95 \%$ (6) of the respondents stated that they could only see three or less tumbling Es with one eye. More than half the respondents $(58.33 \%)$ who read the insertion carefully, had already consulted or were planning to consult an ophthalmologist (Table 2).

The demographic characteristics were analysed to see if any of the demographic variables was associated with noticing the DIY insertion in the newspaper. There were no significant differences in noticing the insertion in respect of age, sex, and occupational status (Table 3). However significant differences were observed with educational level $\left(\chi^{2}-8.6933 ; \mathrm{p}=0.003\right)$.

Younger respondents and males were more likely to have noticed the DIY insertion compared with older respondents or females respectively (Table 3 ). Similarly, students had the highest likelihood of noticing the DIY insertion, but none of these differences were statistically significant. Graduates were 3.83 times more likely to have noticed the DIY insertion compared to those whose educational level was poorer. This difference was statistically significant (OR $-3.83 ; 95 \%$ CI 1.4583 $\left.10.0764 ; \chi^{2}-8.61 ; \mathrm{p}=0.0033\right)$.

The combined circulation of the three newspapers on the 4 days on which the DIY insertion was released was 3170000 . The total cost of the campaign was US $\$ 5500$. The cost of the campaign per copy sold was $\$ 0.002$. Generally, more than one person read a copy of a newspaper and therefore the actual cost of reaching each reader will be even lower. Since

Table 3 Demographic characteristics of respondents noticing communication insertion

\begin{tabular}{|c|c|c|c|c|c|}
\hline Parameters & Freq & $\begin{array}{l}\% \text { noticing } \\
\text { insertion }\end{array}$ & $\chi^{2}$ & $\begin{array}{l}\text { Odds } \\
\text { ratio }\end{array}$ & $95 \% C I$ \\
\hline \multicolumn{6}{|l|}{ Age profile } \\
\hline$\leqslant 25$ years & $13 / 24$ & 54.17 & & 1.0000 & \\
\hline $26-40$ years & $17 / 33$ & 51.51 & & 0.8990 & $0.3103-2.6045$ \\
\hline $41+$ years & $22 / 48$ & 45.83 & $\chi^{2} .5208 ; \mathrm{p} 0.771$ & 0.7160 & $0.2651-1.9334$ \\
\hline Age not recorded/refused & $2 / 20$ & 10.0 & & & \\
\hline \multicolumn{6}{|l|}{ Sex } \\
\hline Male & $25 / 45$ & 55.55 & & 1.0000 & \\
\hline Female & $25 / 63$ & 39.68 & $\chi_{1}^{2}-2.6601 ; \mathrm{p}-0.103$ & 0.5263 & $0.2393-1.1577$ \\
\hline Sex not recorded/refused & $4 / 17$ & 23.53 & & & \\
\hline \multicolumn{6}{|l|}{ Educational status } \\
\hline$<$ Graduate & $8 / 31$ & 25.81 & & 1.0000 & \\
\hline Graduate + & $44 / 77$ & 57.14 & $\chi_{1}^{2}-8.6933$ p 0.003 & 3.8333 & $1.4583-10.0764$ \\
\hline \multicolumn{6}{|l|}{ Status not } \\
\hline recorded/refused & $2 / 17$ & 11.76 & & & \\
\hline \multicolumn{6}{|l|}{ Occupation } \\
\hline \multicolumn{6}{|l|}{ Household work } \\
\hline Students & $11 / 18$ & 61.11 & & 1.2222 & $0.4362-3.4246$ \\
\hline \multicolumn{6}{|l|}{ Business enterprise/self } \\
\hline employed & $14 / 24$ & 58.33 & & 1.8667 & $0.6630-5.2560$ \\
\hline Serving in senior positions & $11 / 23$ & 47.83 & $\chi_{3}^{2} 2.4470 ; \mathrm{p} 0.485$ & 2.0953 & $0.6612-6.6396$ \\
\hline \multicolumn{6}{|l|}{ Occupation not } \\
\hline recorded/refused & $0 / 15$ & 0.00 & & & \\
\hline
\end{tabular}

nearly half the people contacted stated that they had read the DIY insertion, the use of newspapers was highly cost effective.

\section{Discussion}

Most health education strategies adopted in eye care revolve around conventional modes of communication and use of the mass media approaches is still uncommon. This is in spite of the fact that mass media frequently cover health related topics and are the leading source of information on health issues. ${ }^{5}$ Studies have shown that most people place moderate to great trust on health issues reported by mass media. ${ }^{6}$ It is also clear that even a strong persuasive channel, like interpersonal communication, failed to offset the impact of the health press, in the controversy regarding meat consumption after BSE. ${ }^{7}$ Print media campaigns are generally credited with a positive impact on the health seeking behaviour of populations. ${ }^{89}$ However, health programmes can also suffer an adverse impact, as was observed in the case of a newspaper consistently reporting against the vaccination programme, which adversely affected the measles/ mumps/rubella vaccine. ${ }^{10}$ Available evidence shows that using mass media to increase awareness, community participation, and self care in eye care, is a valid method that can be adopted in many countries.

In spite of major advantages with the mass media, strategies focusing on community involvement in health care have never received serious attention. Most developing countries face a resource constraint and therefore innovative programmes, which can effectively motivate communities, and people, to take a proactive role in their health care are urgently required. Breast self examination has been very popular in many countries. It was felt that self examination of vision is a relatively simple procedure to comprehend and act upon and, therefore, it was planned to use this innovative strategy. An effective communication package should be easily comprehensible to people and thereby motivate them to adopt the behavioural change. ${ }^{11}$ In addition, in order to have a long lasting impact, messages must be presented on a regular basis. ${ }^{11}$ How regularly these insertions need to be carried out and at what intervals of time need to be studied as this will determine the total cost of such a campaign. Unfortunately most developing countries do not have enough resources to mount effective communication programmes. Even in the present situation, the innovative communication package could only be marketed because of the support from DFID. It is important that more and more international voluntary agencies, involved in supporting eye care programmes, should specifically earmark funds for developing innovative communication packages.

One medium, which can be targeted for developing communication packages, is the daily newspapers, as this is a very powerful and attractive medium in India. Each week, print media in India reach 242 million Indians. ${ }^{12}$ 
There are an estimated 3740 newspapers published daily in India in all languages, including English. ${ }^{4}$ Fifty million newspapers are purchased in the country every day. ${ }^{12}$ The medium, however, still continues to be predominantly urban based, as $62 \%$ of the 183 million Indians, above the age of 15 years, in urban areas read newspapers or magazines as against only $29 \%$ of the $15+$ populations ( 487 million) residing in rural India. ${ }^{12}$ If the costs per individual reached, by the DIY insertion, in the Indian newspapers, in the present study, were extrapolated to the entire country, the cost effectiveness will be much higher because of the lower costs of campaign costs in the vernacular press in the country.

The impact of the DIY vision testing kit was evaluated by contacting a randomly selected sample of telephone consumers. Every fifth household in the urban areas of India possesses a household telephone. This is mostly limited to the middle and upper socioeconomic segments of the Indian population. ${ }^{13}$ This modality was used because it was important to assess the immediate impact, at an affordable cost, and the telephone seemed to be the best method for this. Telephone interviews are fast, cheap, and can cover a vast geographical area in a short time. ${ }^{14}$ Telephone surveys have been documented to be reliable for estimating health conditions and for dietary recall surveys. ${ }^{14-16}$

It was also observed that respondents with a higher educational attainment tended to pay more attention to the contents of the DIY insertion. Earlier studies also document higher levels of awareness in more literate groups. ${ }^{17}$ The print media should therefore be predominantly directed towards higher socioeconomic strata and the more literate. Since no single strategy can satisfy all the communication needs of the population, a mix of different information, education, and communication (IEC) strategies is required.

The present study shows that for the urban literate populations with a higher level of motivation to act, newspaper insertions can play a major part in changing health seeking behaviour. The present study also shows the cascading effect of such an insertion. Even though only six respondents reported an inability to see three Es clearly, 28 respondents wanted to seek an ophthalmologist's advice. This reflects an increased awareness among the population, regarding the importance of vision, after reading the insertion. Because printed media can be read, clipped out, copied, and passed on, ${ }^{18}$ it has a greater longevity than modalities like posters, banners, radio shows, and television spots. Therefore, increased use of motivational methods, to goad populations to screen their own and their family's vision, is an exciting prospect that eye care providers should consider in the urban milieu of developing countries. This medium coupled with television can reach a significant proportion of the Indian population, if harnessed effectively in the near future.

The authors thank the British Council and the Department for International Development (DFID) for assistance in the design and production of the vision testing kit in the newspapers, as part of the Indo-UK Community Ophthalmology Project.

1 Maibach E, Holtgrave DR. Advances in public health communication. Annu Rev Public Health 1995;16:219-38.

2 Wagman LM. A health department's response to AIDS Condomania: a public education intervention. Can $\mathcal{F}$ Pub Health 1993;84 (Suppl 1):S62-5.

3 Murthy GVS, Kumar S. Health coverage in Indian English newspapers. Hygie 1990;9:34-7.

4 Rahul Singh. Special report: the media in India. The Earth Times 2000:1-17.

5 Grilli R, Freemantle N, Minozzi S, et al. Mass media interventions: effects on health services utilization. Cochrane Database Syst Rev 2000;2:389

6 Johnson T. Shattuck lecture-medicine and the media. $N$ Engl f Med 1998;339:87-92.

7 Verbeke W, Viaene J, Guiot O. Health communication and consumer behavior on meat in Belgium: from BSE until dioxin. F Health Commun 1999;4:345-57.

8 Grilli R. Media have a key role in shaping use of health servGrilli R. Media have a key rol.

9 Savoie I, Kazanjian A, Brunger F. Women, the media, and heart disease: for better or for worse? Int $\mathcal{F}$ Technol Assess Health Care 1999;15:729-37.

10 Mason BW, Donnelly PD. Impact of a local newspaper campaign on the uptake of the measles, mumps and rubella vaccine. $\mathcal{F}$ Epidemiol Community Health 2000;54:473-4.

11 Sitthi Amorn C, Ngamvithayapongse J. The role of the media and communication in improving the use of drugs and other technologies. Int 7 Technol Assess Health Care 1998;14:71-80

12 Swami P. Recording media trends. Frontline 1999;16:105-6. 3 The Gallup Organization. The Gallup India Survey Consumer Report. Gallup.com/poll/reports/india.asp

14 Thomas R, Pardon S. Telephone methods for social surveys. Social Research Update 1994;8:1-6.

15 Starr GJ, Dal Grande E, Taylor AW, et al. Reliability of selfreported behavioral health risk factors in a South Australian telephone survey. Aust NZ F Public Health 1999; 23:528-30.

16 Casey PH, Goolsby SL, Lensing SY, et al. The use of telephone interview methodology to obtain 24-hour dietary recalls. F Am Diet Assoc 1999;11:1406-11.

7 Khoo KL, Liew YM, Tan H, et al. Patients' knowledge and attitude towards treatment and control of hypertension: nation-wide telephone survey conducted in Malaysia. Med 7 Malaysia 1999;54:37-46.

18 Austin LS, Husted K. Cost effectiveness of television, radio and print media programs for public mental health education. Psychiatr Serv 1998;49:808-11. 